FERMILAB-Conf-89/180-T

August 1989

\title{
THE STRUCTURE OF GLUON RADIATION IN QCD ${ }^{1}$
}

\author{
Stephen PARKE \\ Fermi National Accelerator Laboratory P.O. Box 500, Batavia, IL 60510. \\ and \\ Michelangelo MANGANO \\ Istituto Nazionale di Fisica Nucleare \\ S. Piero a Grado, Pisa, ITALY
}

\begin{abstract}
For massless QCD the hard scattering amplitudes are naturally written in terms of the dual color expansion. Here I present this expansion for purely gluonic processes and processes involving quark-antiquark pairs and gluons. The properties of the sub-amplitudes as well as explicit algebraic expressions are given for a number of these processes. Also, I demonstrate how to recover massless QED amplitudes from the dual expansion of massless QCD.
\end{abstract}

\section{Introduction}

In perturbative QCD the calculation of multi-gluon scattering amplitudes, even at tree level, is very challenging. Part of the reason for the difficulty is that up to recently there has been no systematic way to efficiently identify the appropriate gauge invariant subsets of the full amplitude. Here I summarize what has been discovered on how to make this division ${ }^{[1]}$. By insuring that the gauge invariant subsets are invariant under cyclic permutations of the external gluons tremendous cancellations occur at the amplitude level and the sub-amplitudes so defined have remarkable factorization properties. The generalization to $\mathrm{QCD}$ processes involving one or more quark-antiquark pairs is also given.

\footnotetext{
"Invited talk presented by SP at "The Workshop on QED Structure Functions", University of Michigan, Ann Arbor, MI; May 22-25,1989.
} 


\section{Duality and Gauge Invariance}

Consider an $S U(N)$ Yang-Mills theory, then at tree level in perturbation theory, any vector particle scattering amplitude, with colors $a_{1}, a_{2} \ldots a_{n}$, external momenta $p_{1}, p_{2} \ldots p_{n}$ and helicities $\epsilon_{1}, \epsilon_{2} \ldots \epsilon_{n}$, can be written as

$$
\mathcal{M}_{n g}=\sum_{p e r m^{\prime}} \operatorname{tr}\left(\lambda^{a_{1}} \lambda^{a_{2}} \ldots \lambda^{a_{n}}\right) m\left(p_{1}, \epsilon_{1} ; p_{2}, \epsilon_{2} ; \cdots ; p_{n}, \epsilon_{n}\right),
$$

where the sum, perm' ${ }^{\prime}$, is over all (n-1)! non-cyclic permutations of $1,2, \ldots, n$ and the $\lambda$ 's are the matrices of the symmetry group in the fundamental representation. This expansion is known as the dual expansion because of the invariance of the sub-amplitudes under cyclic permutations ${ }^{[1]}$.

The proof that one can always make this expansion is very simple using the identities $\left[\lambda^{a}, \lambda^{b}\right]=i \sqrt{2} f_{a b c} \lambda^{c}$ and $\operatorname{tr}\left(\lambda^{a} \lambda^{b}\right)=\delta^{a b}$. In any tree level Feynman diagram, replace the color structure function at some vertex using

$$
f_{a b c}=-(i / \sqrt{2}) \operatorname{tr}\left(\lambda^{a} \lambda^{b} \lambda^{c}-\lambda^{c} \lambda^{b} \lambda^{a}\right) .
$$

Now each leg attached to this vertex has a $\lambda$ matrix associated with it. At the other end of each of these legs there is either another vertex or this is an external leg. If there is another vertex, use the $\lambda$ associated with this internal leg to write the structure function of this vertex $f_{\text {cde }} \lambda^{c}$ as $-i\left[\lambda^{d}, \lambda^{e}\right] / \sqrt{2}$. Continue this processes until all vertices have been treated in this manner. Then this Feynman diagram has been placed in the form of eqn(2.1). Repeating this procedure for all Feynman diagrams for a given process completes the proof.

The sub-amplitudes $m(1,2, \ldots, n) \equiv m\left(p_{1}, \epsilon_{1} ; p_{2}, \epsilon_{2} ; \ldots p_{n}, \epsilon_{n}\right)$ of eqn(2.1) satisfy a number of important properties and relationships.

(1) $m(1,2, \ldots, n)$ is gauge invariant.

(2) $m(1,2, \ldots, n)$ is invariant under cyclic permutations of $1,2, \ldots, n$

(3) $m(n, n-1, \ldots, 1)=(-1)^{n} m(1,2, \ldots, n)$

(4) The Dual Ward Identity:

$$
\begin{array}{r}
m(1,2,3, \ldots, n)+m(2,1,3, \ldots, n)+m(2,3,1, \ldots, n) \\
+\cdots+m(2,3, \ldots, 1, n)=0
\end{array}
$$

(5) Factorization of $m(1,2, \cdots, n)$ in the soft, collinear and multi-gluon pole limits.

(6) Incoherence to leading order in number of colors:

$$
\sum_{\text {color. }}\left|\mathcal{M}_{n g}\right|^{2}=N^{n-2}\left(N^{2}-1\right) \sum_{\text {perm }}\left\{|m(1,2, \cdots, n)|^{2}+\mathcal{O}\left(N^{-2}\right)\right\} .
$$


This set of properties for the sub-amplitudes, we will refer to as duality and the expansion in terms of these dual sub-amplitudes the dual expansion. Properties (1) and (2) can be seen directly from the properties of linear independence, for arbitrary $\mathrm{N}$, and invariance under cyclic permutations of $\operatorname{tr}\left(\lambda^{1} \lambda^{2} \ldots \lambda^{n}\right)$. Whereas (3) and (4) follow by studying the sum of Feynman diagrams which contribute to each sub-amplitude. The sum of Feynman diagrams which make the Dual Ward Identity is such that each diagram is paired with another with opposite sign so that the combination contained in eqn(2.3) trivially vanishes. Property (5) will be discussed in great detail in section IV and the incoherence to leading order in the number of colors (6) follows from the color algebra of the $S U(N)$ gauge group.

To the string theorist this expansion and the duality properties (1) to (6), see [2], are quite familar since the string amplitude, in the zero slope limit, reproduces the Yang-Mills amplitude on mass shell [3]. Each sub-amplitude is then represented by the zero slope limit of a string diagram, and the sub-amplitude could be obtained by using the usual Koba-Nielsen formula [4]. The traces of $\lambda$ matrices are just the Chan-Paton factors ${ }^{[5]}$. For the string amplitude the properties (1) through (6) are satisfied even before the zero slope limit is taken. Also from the string diagrams it is simple to see which Feynman diagrams contribute to a given sub-amplitude, e.g. Fig. 1. The coefficients for the contributing diagrams are obtained by the procedure developed earlier in this section for rewriting the color factors. The relationship between the string diagram and our dual sub-amplitudes suggests that a Yang-Mills amplitude expressed in terms of these dual sub-amplitudes will assume a particularly simple form.
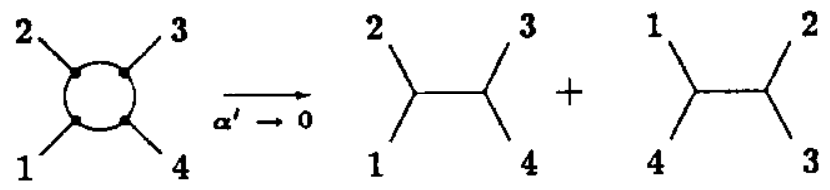

Figure 1: The zero-slope limit of the four gluon string diagram in terms of Feynman diagrams (tri-gluon couplings only).

The gauge invariance and properties under cyclic and reverse permutations allows the calculation of far fewer than the $(n-1)$ ! sub-amplitudes that appear in the dual expansion. In fact the number of sub-amplitudes that are needed is just the number of different orderings of positive and negative helicities around a circle. Of course some of the sub-amplitudes vanish because of the partial helicity 
conservation of tree level Yang-Mills and others are simply related to one another through the properties (2) through (4).

\section{Spinor Products}

To evaluate the sub-amplitudes we have used the helicity basis for the polarization vectors which was introduced by $\mathrm{Xu}$, Zhang and Chang[6]. This technique requires the introduction of the concept of a spinor product of two light-like momentum vectors. We define the following symbols for the chiral spinors associated with the light-like momenta, $p_{i}$, and their spinor products:

$$
\begin{aligned}
& |i \pm\rangle \equiv \frac{1}{2}\left(1 \pm \gamma_{5}\right) u\left(p_{i}\right), \quad\langle i \pm| \equiv \bar{u}\left(p_{i}\right) \frac{1}{2}\left(1 \mp \gamma_{5}\right) \\
& \langle i j\rangle=\langle i-\mid j+\rangle, \quad[i j]=\langle i+\mid j-\rangle=\operatorname{sign}\left(p_{i}^{0} p_{j}^{0}\right)\langle j i\rangle^{*} .
\end{aligned}
$$

The important properties of these spinor products that will be needed in this paper are that both $\langle i j\rangle$ and $[i j]$ are odd under interchange of $i$ and $j$ and are complex square roots of the Lorentz invariant $S_{i j} \equiv\left(p_{i}+p_{j}\right)^{2}$;

$$
\begin{aligned}
& \langle i j\rangle \equiv \sqrt{\left|S_{i j}\right|} \exp \left(i \phi_{i j}\right) \\
& {[i j] \equiv \sqrt{\left|S_{i j}\right|} \exp \left(i \bar{\phi}_{i j}\right)}
\end{aligned}
$$

If both momenta having positive energy, the phase factor $\phi_{i j}$ is defined, in a popular representation of the gamma matrices, by

$$
\begin{aligned}
\cos \phi_{i j} & =\frac{\left(p_{i}^{1} p_{j}^{+}-p_{j}^{1} p_{i}^{+}\right)}{\sqrt{p_{i}^{+} p_{j}^{+}}} \\
\sin \phi_{i j} & =\frac{\left(p_{i}^{2} p_{j}^{+}-p_{j}^{2} p_{i}^{+}\right)}{\sqrt{p_{i}^{+} p_{j}^{+}}} .
\end{aligned}
$$

Where $p^{ \pm}=\left(p^{0} \pm p^{3}\right)$ and since all $p_{i}^{2}=0$ the spinor product for this representation of gamma matrices is undefined for a momentum vector in the minus 3 direction. If one or more of the momenta in $\langle i j\rangle$ have negative energy, $\phi_{i j}$ is calculated with minus the momenta with negative energy and then $n \pi / 2$ is added to $\phi_{i j}$ where $\mathrm{n}$ is the number of negative momenta in the spinor product. The associated phase factor, $\tilde{\phi}_{i j}$, for $[i j]$ can be found using equation (3.2) or calculated from $S_{i j}$ using the identity $S_{i j} \equiv\langle i j\rangle[j i]$. 


\section{Factorization Properties}

The most important and remarkable properties of the Yang-Mills dual subamplitudes are their factorization properties, whose origin can be traced back to the string picture. In this section we give the factorization properties of the gluon sub-amplitudes in

(1) the soft gluon limit,

(2) when two gluons become collinear and

(3) when three gluons add to form an on mass-shell gluon i.e. on the three gluon pole.

For arbitrary $n$-gluon scattering these factorization properties of the sub-amplitudes will extend up to factorization on the $[n / 2]$-gluon poles.

First, we consider the soft gluon limit. Consider the sub-amplitudes when gluon 1 has an energy which is small compared to all the other energies in the process. Then the gluon sub-amplitudes must satisfy

$$
\begin{aligned}
& m\left(1^{+}, 2 \ldots, n\right) \stackrel{1^{+} \text {.oft }}{\longrightarrow}\left\{\frac{g\langle n 2\rangle}{\langle n 1\rangle\langle 12\rangle}\right\} m(2,3 \ldots, n) \\
& m\left(1^{-}, 2 \ldots, n\right) \stackrel{1-. \text {.oft }}{\longrightarrow}\left\{\frac{g[n 2]}{[n 1][12]}\right\} m(2,3 \ldots, n) .
\end{aligned}
$$

The factors in braces are square roots of the eikonal factor

$$
\frac{g^{2}\left(p_{n} \cdot p_{2}\right)}{\left(p_{n} \cdot p_{1}\right)\left(p_{1} \cdot p_{2}\right)}
$$

This soft gluon factorization and the incoherence of these sub-amplitudes to leading order in the number of colors, $\mathrm{N}$, leads to the soft gluon factorization of the full matrix element squared as proposed by Bassetto, Ciafaloni and Marchesini [7],

$$
\sum_{\text {color: }}\left|\mathcal{M}_{n g}\right|^{2} \stackrel{1}{\longrightarrow} \stackrel{\text { oft }}{\longrightarrow} \sum_{i j}\left(\frac{g^{2}\left(p_{i} \cdot p_{j}\right)}{\left(p_{i} \cdot p_{1}\right)\left(p_{1} \cdot p_{j}\right)}\right)\left|A_{i j}(2, \cdots, n)\right|^{2} .
$$

In the limit when two gluons become collinear, Altarelli and Parisi [8] demonstrated that the double poles associated with this collinear pair do not appear in the full amplitude squared i.e. there is a cancellation of one power of the propagator of the sum of the two collinear gluons. This cancellation occurs at the amplitude level rather than the square of the amplitude in this dual formulation. Therefore the squared sub-amplitudes diverge no more rapidly than a single power of the propagator for the collinear gluons, this is the Altarelli and Parisi 
observation. The origin of this behaviour of the dual sub-amplitudes stems from the factorization properties of string amplitudes.

To demonstrate this square root divergence of the sub-amplitudes in the collinear limit, consider the case when the momenta of particles 1 and 2 become parallel. Let $1 \rightarrow z P$ and $2 \rightarrow(1-z) P$ with $P^{2}=0$, and $z$ is the momentum fraction of particle 1 . Then the sub-amplitudes become

$$
\begin{aligned}
& m\left(1^{+}, 2^{+}, 3, \ldots\right) \stackrel{1^{+} \|_{2}^{+}}{\longrightarrow}\left\{\frac{i g[12]}{\sqrt{z(1-z)}}\right\} \frac{-i}{S_{12}} m\left(P^{+}, 3, \ldots\right) \\
& m\left(1^{+}, 2^{-}, 3, \ldots\right) \stackrel{1^{+} \|^{2-}}{\longrightarrow}\left\{\frac{i g z^{2}(12\rangle}{\sqrt{z(1-z)}}\right\} \frac{-i}{S_{12}} m\left(P^{+}, 3, \ldots\right) \\
& +\left\{\frac{i g(1-z)^{2}[12]}{\sqrt{z(1-z)}}\right\} \frac{-i}{S_{12}} m\left(P^{-}, 3, \ldots\right) \\
& m\left(1^{-}, 2^{-}, 3, \ldots\right) \stackrel{1^{-} \|^{-}}{\longrightarrow}\left\{\frac{i g(12\rangle}{\sqrt{z(1-z)}}\right\} \frac{-i}{S_{12}} m\left(P^{-}, 3, \ldots\right) .
\end{aligned}
$$

Note that either $\langle 12\rangle$ or [12] appears in the numerator of each term. Also, it is useful to interpret the factor in braces as the "three gluon sub-amplitude" in the limit when two gluons become collinear. This three gluon sub-amplitude has the square root suppression of the pole as well as having the square root of the appropriate Altarelli-Parisi gluon-fusion function. From this result and the incoherence of the sub-amplitudes in the square of the matrix element the standard results of Altarelli and Parisi are obtained in a simple manner.

The sub-amplitudes also factorize in the three particle channel; here let $P=$ $1+2+3$, then as $P^{2} \rightarrow 0$ it is easy to see that

$$
m(1,2,3,4,5,6) \rightarrow m(1,2,3,-P) \frac{-i}{P^{2}} m(P, 4,5,6)
$$

for the helicity structure three positive and three negative. Since helicity is conserved in the four gluon process, the helicity of the intermediate gluon is determined for this helicity structure and the four positive - two negative helicity sub-amplitude has no three particle poles.

Of course the full matrix element must also factorize. This is trivial in Feynman diagram language but here it is not so obvious because of the way we have added diagrams together. The color factors almost factorizes for an $S U(N)$ gauge group,

$$
\begin{aligned}
\operatorname{tr}\left(\lambda^{1} \lambda^{2} \ldots \lambda^{n}\right)=\quad \sum_{x} \operatorname{tr}\left(\lambda^{1} \ldots \lambda^{m} \lambda^{x}\right) \operatorname{tr}\left(\lambda^{x} \lambda^{m+1} \ldots \lambda^{n}\right) \\
+\frac{1}{N} \operatorname{tr}\left(\lambda^{1} \ldots \lambda^{m}\right) \operatorname{tr}\left(\lambda^{m+1} \ldots \lambda^{n}\right) .
\end{aligned}
$$


This "factorization" property of the traces follows from the identity

$$
\sum_{a} \lambda_{i j}^{a} \lambda_{k l}^{a}=\left(\delta_{i l} \delta_{j k}-\frac{1}{N} \delta_{i j} \delta_{k l}\right) .
$$

The $1 / N$ term could destroy the full factorization, but it does not. Terms proportional to $1 / N$ vanish at the pole because of the Dual Ward Identity for the sub-amplitudes. Therefore, all the gluon amplitudes discussed in this paper satisfy, as expected, the factorization property

$$
\mathcal{M}_{n+n^{\prime}} \rightarrow \sum \mathcal{M}_{n+1} \frac{-i}{P^{2}} \mathcal{M}_{n^{\prime}+1}
$$

as $P^{2} \rightarrow 0$ for $n, n^{\prime} \geq 2$. The sum is over the color and helicity of the intermediate state.

\section{Pure Gluon Amplitudes}

For four gluon scattering only the helicity conserving amplitudes are non zero. Using the convention that all particles are labelled with their helicities and momenta as if they were outgoing, i.e. the incoming particles have negative energies, the helicity conserving sub-amplitudes are given by

$$
\begin{aligned}
m_{2+2}(1,2,3,4) & =-i g^{2} \frac{\langle I J\rangle^{2}[K L]^{2}}{S_{12} S_{23}} \\
& =i g^{2} \frac{\langle I J\rangle^{4}}{\langle 12\rangle\langle 23\rangle\langle 34\rangle\langle 41\rangle} .
\end{aligned}
$$

The momenta $I$ and $J(K$ and $L$ ) in the numerator are the momenta of the negative (positive) helicity gluons independent of their ordering in the sub-amplitude, whereas the order of the spinor products in the denominator is only determined by the order of the momenta in the sub-amplitude. Using the properties of the spinor product is simple to demonstrate that eqn(5.1) satisfies the four particle Dual Ward Identity (2.3).

In squaring the four gluon amplitude and summing over colors the $\mathcal{O}\left(\mathrm{N}^{-2}\right)$ terms in eqn(2.4) can be shown to vanish by using only the general properties, especially the Dual Ward Identity, of the sub-amplitudes. Therefore,

$$
\sum_{\text {color. }}\left|\mathcal{M}_{4 g}\right|^{2}=N^{2}\left(N^{2}-1\right) \sum_{\text {perm }}|m(1,2,3,4)|^{2},
$$

and the square of each sub-amplitude is very simple because the spinor product is the square root of twice the dot product. The final result is the standard four 
gluon matrix element squared.

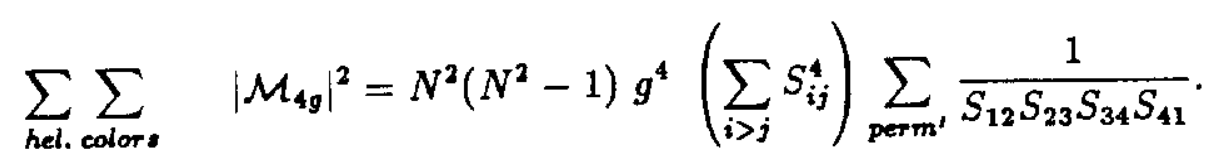

Here we have not averaged over incoming helicities or colors.

For five gluon scattering only those Feynman diagrams, or part there of, with color structure the same as the diagrams of Fig. 2 contribute to the $m(1,2,3,4,5)$ sub-amplitude.

Again, it is a straight forward, simple calculation [1] to show that the only nonzero sub-amplitudes have either two or three negative helicity gluons and that the three positive - two negative helicity sub-amplitude is given by

$$
m_{3+2-}(1,2,3,4,5)=i g^{3} \frac{\langle I J\rangle^{4}}{\langle 12\rangle\langle 23\rangle\langle 34\rangle\langle 45\rangle\langle 51\rangle} .
$$

Where $I$ and $J$ are again the momenta of the negative helicity gluons and the denominator ordering is determined by the order of the momenta in the subamplitude. The two positive - three negative helicity amplitude is obtained from this last equation by complex conjugation. By using the Fierz properties of the spinor product it is easy to demonstrate that eqn(5.4) satisfies the five particle Dual Ward Identity, eqn(2.3).
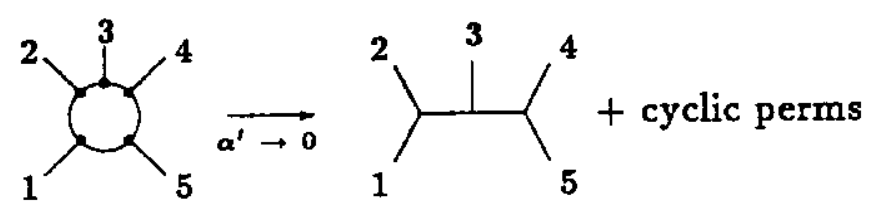

Figure 2: The zero-slope limit of the five gluon string diagram in terms of Feynman diagrams (tri-gluon couplings only).

Again, the general properties of the sub-amplitude can be used to show that the $\mathcal{O}\left(N^{-2}\right)$ terms in eqn(2.4) vanish for the five gluon process giving the following standard result [9] that

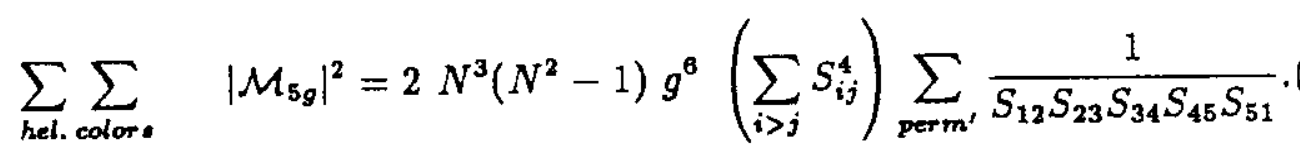


Here we have not averaged over incoming helicities or colors.

For the six gluon process only those Feynman diagrams, or part there of, with the same color structure as the diagrams of Fig. 3 contribute to the $m(1,2,3,4,5,6)$ sub-amplitude. Then, by using the appropriate reference momenta for the polarization vectors it is easy to see that the only non-zero subamplitudes are those with four positive - two negative, two positive - four negative and three positive - three negative helicities. After a lengthy calculation we have obtained the following expressions for the six gluon sub-amplitudes.

The sub-amplitudes for the four positive - $t$ wo negative helicity processes are a straight forward generalization of the four and five-gluon sub-amplitudes;

$$
m_{4+2-}(1,2,3,4,5,6)=i g^{4} \frac{\langle I J\rangle^{4}}{\langle 12\rangle\langle 23\rangle\langle 34\rangle\langle 45\rangle\langle 56\rangle\langle 61\rangle} .
$$

Again, I and $J$ represent the momenta of the negative helicity gluons. Different permutations can be obtained as before by keeping fixed the numerator and permuting the momenta in the denominator. The two positive - four negative helicity sub-amplitude is obtained from eqn(5.6) by complex conjugation.
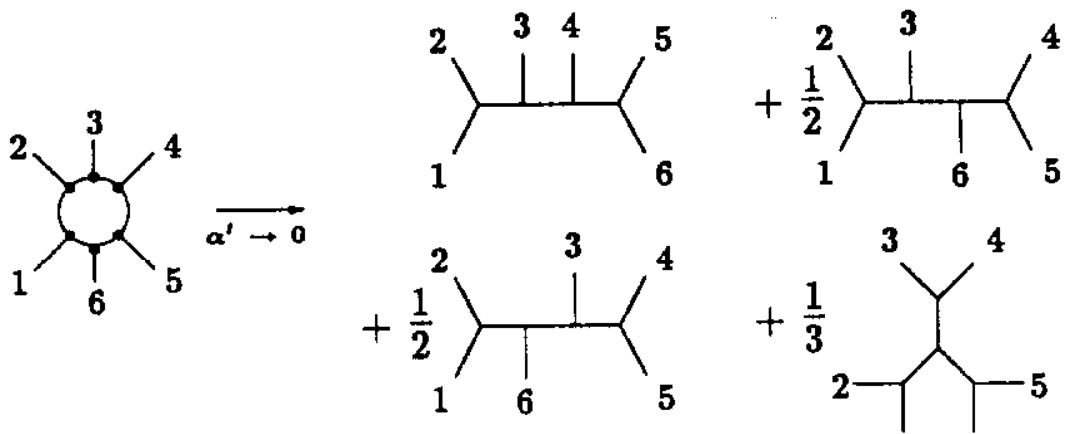

6

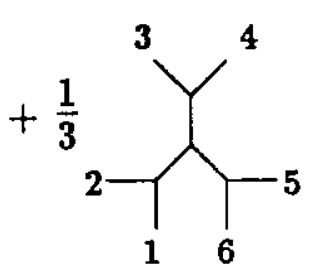

+ cyclic perms

Figure 3: The zero-slope limit of the six gluon string diagram in terms of Feynman diagrams (tri-gluon couplings only).

The three positive - three negative helicity sub-amplitudes are not as simple. To exhibit the factorization on the three particle channels these sub-amplitudes are

$$
\begin{aligned}
m_{3+3-}(1,2,3,4,5,6) & =i g^{4}\left[\frac{\alpha^{2}}{t_{123} S_{12} S_{23} S_{45} S_{58}}+\frac{\beta^{2}}{t_{234} S_{23} S_{34} S_{58} S_{61}}\right. \\
& \left.+\frac{\gamma^{2}}{t_{345} S_{34} S_{45} S_{61} S_{12}}+\frac{t_{123} \beta \gamma+t_{234} \gamma+t_{345} \alpha \beta}{S_{12} S_{23} S_{34} S_{45} S_{56} S_{81}}\right]
\end{aligned}
$$


where the $t_{i j k} \equiv\left(p_{i}+p_{j}+p_{k}\right)^{2}=S_{i j}+S_{j k}+S_{k i}$. The coefficients $\alpha, \beta$ and $\gamma$ for the three distinct orderings of the helicities are given in Table I. With this representation it is a simple exercise to show that these sub-amplitudes factorize on the three particle pole into a product of two four particle sub-amplitudes, eqn(5.1), times the three particle propagator.

\section{Table I}

Coefficients for the $m_{3+3}-S u b$-amplitudes: where $\langle I|K| J\rangle \equiv\langle I+|K \cdot \gamma| J+\rangle$, which is linear in $\mathrm{K}$ and if $K^{2}=0$ is given by $[I K]\langle K J\rangle$.

\begin{tabular}{|l||c|c|c|}
\hline \hline & $1^{+2^{+} 3^{+} 4^{-5^{-}} 6^{-}}$ & $1^{+2^{+} 3^{-} 4^{+5^{-}} 6^{-}}$ & $1^{+2^{-} 3^{+} 4^{-5^{+}} 6^{-}}$ \\
& $X=1+2+3$ & $Y=1+2+4$ & $Z=1+3+5$ \\
\hline \hline$\alpha$ & 0 & $-[12]\langle 56\rangle\langle 4|Y| 3\rangle$ & {$[13]\langle 46\rangle\langle 5|Z| 2\rangle$} \\
\hline$\beta$ & {$[23]\langle 56\rangle\langle 1|X| 4\rangle$} & {$[24]\langle 56\rangle\langle 1|Y| 3\rangle$} & {$[51]\langle 24\rangle\langle 3|Z| 6\rangle$} \\
\hline$\gamma$ & {$[12]\langle 45\rangle\langle 3|X| 6\rangle$} & {$[12]\langle 35\rangle\langle 4|Y| 6\rangle$} & {$[35]\langle 62\rangle\langle 1|Z| 4\rangle$} \\
\hline
\end{tabular}

The six gluon sub-amplitudes satisfy the three distinct Dual Ward Identities obtained from the following equation

$$
\begin{aligned}
m(1,2,3,4,5,6) & +m(2,1,3,4,5,6)+m(2,3,1,4,5,6) \\
+ & m(2,3,4,1,5,6)+m(2,3,4,5,1,6)=0
\end{aligned}
$$

using the helicity ordering of the first term as either $m(1+, 2+, 3+, 4+, 5-, 6-)$, $m(1+, 2+, 3+, 4-, 5-, 6-)$ or $m(1+, 2-, 3+, 4-, 5+, 6-)$. These three Identities are extremely powerful and relate sub-amplitudes with different orderings of the helicities.

Given the simplicity of the sub-amplitudes with two negative helicities and all the others positive, equations (5.1), (5.4) and (5.6), it is obvious that the generalization to arbitrary $n$ is

$$
m_{(n-2)+2-}(1,2, \ldots, n)=i g^{n-2} \frac{\langle I J\rangle^{4}}{\langle 12\rangle\langle 23\rangle \cdots\langle n 1\rangle}
$$

where once again $I$ and $J$ are the momenta of the negative helicity gluons. Apart from this being the natural square root of the expression given by Parke and Taylor [10], [7], it also satisfies the Dual Ward Identity for arbitrary $n$.

The complete square of the six-gluon amplitude, including the non-leading color terms is

$$
\sum_{\text {color, }}\left|\mathcal{M}_{6 g}\right|^{2}=
$$




$$
\begin{aligned}
& N^{4}\left(N^{2}-1\right) \sum_{\text {perm }} \mid \\
& +\frac{1}{N^{2}}\left(m^{*}(1,2,3,4,5,6)[m(1,3,5,2,6,4)\right. \\
& \quad+m(1,3,6,4,2,5)+m(1,4,2,6,3,5)]+c . c) .
\end{aligned}
$$

Note that the sub-amplitudes add incoherently to leading order in the number of colors and the simplicity of the non-leading color terms is achieved by the properties of the sub-amplitudes, especially the Dual Ward Identity equation (2.3). This result together with the expressions for the sub-amplitudes, eqn(5.6) and (5.7), can be used to calculate the matrix element squared by evaluating the sub-amplitudes as complex numbers. Owing to the simplicity of the subamplitudes and the simplicity of the leading and non-leading terms in the number of colors this method of calculation is appreciable faster than previous numerical algorithms [11], [12].

The ordering of the gluons in the non-leading color terms is of particular import. These terms are the only possible ones which have no two or three particle propagators in common with the original ordering $(1,2,3,4,5,6)$ and as such are less singular in the collinear limit than the leading part in $N$. In fact the non-leading color terms are finite in the collinear limit so that in this limit they are completely irrelevant compared to the leading color terms. Also by comparing numerically the leading to non-leading pieces for $\mathrm{N}=3$, the non-leading terms contribute in general only a few percent to the total cross-section. This result is even true in the soft gluon limit. Therefore the non-leading terms can be ignored given that this calculation is only to tree level, and the other uncertainties in any Monte Carlo application are much larger than this uncertain. The smallness of the non-leading color terms and the fact that the leading color terms are just the squares of the simple sub-amplitudes implies that the square of this matrix element is easy to obtain.

\section{The Addition of Quarks}

The dual basis is modified by the addition of a quark-antiquark pair in the scattering amplitude in the following way. Consider a quark and an antiquark with colors $\alpha$ and $\bar{\alpha}$ respectively then we write the amplitude as

$$
\mathcal{M}_{\overline{q q n g}}=\sum_{p \in r m}\left(\lambda^{a_{1}} \lambda^{a_{2}} \ldots \lambda^{a_{n}}\right)_{\alpha \bar{\alpha}} m_{\bar{q} q}\left(p_{\bar{q}}, \epsilon_{\bar{q}} ; p_{q}, \epsilon_{q} ; p_{1}, \epsilon_{1} ; \cdots ; p_{n}, \epsilon_{n}\right)(6.1)
$$

where the sum, perm, is over all $n$ ! permutation of the gluons. This expansion of the quark amplitude in terms of this color basis is well known and in particular 
was used by Kunszt in Reference [12]. We will call the color basis in Equation (6.1) the quark dual basis ${ }^{[13],[14] .}$

For the amplitude squared, we have an expression very similar to Equation (2.4):

$$
\sum_{\text {color. }}\left|\mathcal{M}_{q q n g}\right|^{2}=N^{n-3}\left(N^{2}-1\right) \sum_{\{1, \ldots, n\}}\left\{\left|m_{\bar{q} q}(\bar{q}, q, 1, \cdots, n)\right|^{2}+\mathcal{O}\left(N^{-2}\right)\right\} .
$$

Notice however the change in the exponent of the leading power of $N$. The explicit form of the sub-leading terms for $n=2,3,4$ is given in reference [14].

As in the pure gluon case the amplitudes with all particles or all but one particle having the same helicity vanishes at tree level. Also the quark and the antiquark must have opposite helicity or otherwise the amplitude vanishes from chirality conservation in massless QCD. The amplitudes with one gluon the same helicity as the quark or antiquark and all other gluons having the opposite helicity have simple expressions:

$$
\begin{aligned}
& m_{\bar{q} q}\left(\bar{q}^{+}, q^{-}, g_{1}^{+}, \ldots, g_{I}^{-}, \ldots, g_{n}^{+}\right)=i g^{n} \frac{\langle\bar{q} I\rangle\langle q I\rangle^{3}}{\langle\bar{q} q\rangle\langle q 1\rangle\langle 12\rangle \cdots\langle n \bar{q}\rangle} . \\
& m_{q q}\left(\bar{q}^{-}, q^{+}, g_{1}^{+}, \ldots, g_{I}^{-}, \ldots, g_{n}^{+}\right)=i g^{n} \frac{\langle\bar{q} I\rangle^{3}\langle q I\rangle}{\langle\bar{q} q\rangle\langle q 1\rangle\langle 12\rangle \cdots\langle n \bar{q}\rangle} .
\end{aligned}
$$

Other amplitudes are more complex, e.g. the general form of the quarkantiquark four gluon amplitude has the following pole structure as dictated by duality ${ }^{[2]}$ :

$$
\begin{aligned}
m_{q q}\left(\bar{q}, q, g_{1}, g_{2}, g_{3}, g_{4}\right) & =i g^{4}\left[\frac{P_{1}}{t_{\bar{q} 1} S_{\bar{q} q} S_{q 1} S_{23} S_{34}}+\frac{P_{2}}{t_{q 12} S_{q 1} S_{12} S_{34} S_{4 \bar{q}}}\right. \\
& \left.+\frac{P_{3}}{t_{123} S_{12} S_{23} S_{4 \bar{q}} S_{\bar{q}}}+\frac{P_{q}}{S_{q q} S_{q 1} S_{12} S_{23} S_{34} S_{4 \bar{q}}}\right]
\end{aligned}
$$

The numerators $P_{i}$ are complicated and I refer you to reference [14] for explicit expressions for these quantities. The sub-amplitudes defined by equation (6.1) have similar properties to the purely gluonic ones in the soft, collinear and multiparticle pole limits.

To construct the QED results from the non-abelian amplitudes all that is needed is to replace $\lambda_{\alpha \alpha}$. in equation (6.1) by $\delta_{\alpha \bar{\alpha}}$, for details see reference [15]. For example one of the helicity amplitudes for the amplitudes involving an electron, positron and $n$ photons can be written as

$$
\begin{aligned}
\mathcal{M}\left(\bar{e}^{+}, e^{-} ; \gamma_{1}^{+}, \ldots, \gamma_{\bar{I}}, \ldots, \gamma_{n}^{+}\right) & =i e_{0}^{n} \frac{\langle\bar{e} I\rangle\langle e I\rangle^{3}}{\langle\bar{e} e\rangle} \sum_{\{1, \ldots, n\}} \frac{1}{\langle e 1\rangle\langle 12\rangle \cdots\langle n \bar{e}\rangle} \\
& =i e_{0}^{n} \frac{\langle\bar{e} I\rangle\langle e I\rangle^{3}}{\langle\bar{e} e\rangle^{2}} \prod_{j} \frac{\langle\bar{e} e\rangle}{\langle e j\rangle\langle j \bar{e}\rangle} .
\end{aligned}
$$


In this example one can see that for the abelian amplitude the photons are emitted independently of each other whereas for the non-abelian amplitude there is a correlation between the emitted gluons.

To extend this quark dual basis to more than one quark antiquark pair I refer you to the paper by Mangano [15], and here I briefly sketch the color basis. For two quark-antiquark pairs of different flavors with colors $\alpha, \alpha^{\prime}$ and $\bar{\alpha}, \bar{\alpha}^{\prime}$ the dual color expansion is

$$
\begin{aligned}
\mathcal{M}_{\bar{q} q \bar{q}^{\prime} q^{\prime} n g}=\sum_{\{\sigma, \tau\}}\left(\prod_{\sigma} \lambda\right)_{\alpha \alpha^{\prime}}\left(\prod_{\tau} \lambda\right)_{\alpha^{\prime} \bar{\alpha}} m_{\Phi q \bar{q}^{\prime} q^{\prime}}^{x}(\sigma, \tau) \\
+\frac{1}{N}\left(\prod_{\sigma} \lambda\right)_{\alpha \bar{\alpha}}\left(\prod_{\tau} \lambda\right)_{\alpha^{\prime} \alpha^{\prime}} m_{\Phi q \Phi^{\prime} q^{\prime}}^{0}(\sigma, \tau)
\end{aligned}
$$

where the sum is over all partitions, $\{\sigma, \tau\}$, of all permutations of the $\mathrm{n}$ gluons. The first term is the contribution in which the color flow connects $\alpha$ to $\bar{\alpha}^{\prime}$ and $\alpha^{\prime}$ to $\bar{\alpha}$ whereas the second term comes from the color flow connecting $\alpha$ to $\bar{\alpha}$ and $\alpha^{\prime}$ to $\bar{\alpha}^{\prime}$. For two quark pairs of the same flavor one must add $\mathcal{M}_{\bar{q} q^{\prime} q^{\prime} q r g}$ to $\mathcal{M}_{q q q^{\prime} q^{\prime} n g}$. Similar factorization properties to $\mathrm{m}_{q q}$ also hold for $\mathrm{m}_{\bar{q} q q^{\prime} q^{\prime}}^{\pi}$ and $\mathrm{m}_{q q q^{\prime} q^{\prime}}^{0}$.

Finally, the simplicity of the helicity amplitudes with two partons having opposite helicity from all the others, eqn(5.9) and (6.3) has start an industry of approximation methods for these multi-parton processes, see reference [16] for details.

\section{Conclusion}

Here we have demonstrated that the dual color basis is the natural one for QCD amplitudes. The sub-amplitudes defined by this basis have remarkable factorization properties and are quite simple given the complexity of processes of QCD. From purely gluonic processes to processes involving quarks and other vector particles, this basis displays the underlying physics of the process.

\section{References}

[1] M. Mangano, S. Parke and Z. Xu, in Proc. of "Les Rencontres de Physique de la Vallee d'Aoste", La Thuile, Italy, (1987), ed. M. Greco, Editions Frontières, p.513;

M. Mangano, S. Parke and Z. Xu, Nucl. Phys. B298 (1988) 653;

F. A. Berends and W. Giele, Nucl. Phys. B294 (1987) 700.

[2] See for example M. Jacob and S. Mandelstam's lectures contained in 'Lectures on Elementary Particles and Quantum Field Theory', Brandeis 1970, S. 
Deser, M. Grisaru and H. Pendleton eds., MIT Press and reference contained in these lectures.

[3] For a comprehensive review see: J. Schwarz, Phys. Rep. 89 (1982), 224.

[4] Z. Koba and H. B. Nielsen, Nucl. Phys. B10 (1969), 633; B12 (1969), 517: Evaluation of the integrals and taking the zero slope limit is extremely tedious for more than five external particles so the sub-amplitudes were evaluated using Feynman perturbation theory.

[5] J. Paton and Chan Hong-Mo, Nucl. Phys. B10 (1969) 519.

[6] Z. Xu, Da-Hua Zhang and L. Chang, Tsinghus University Preprints, Beijing, The People's Republic of China, TUTP-84/4, TUTP-84/5, TUTP-84/6 and Nucl. Phys. B291 (1987) 392.

[7] A. Bassetto, M. Ciafaloni and P. Marchesini, Phys. Rep. 100 (1983), 201.

[8] G. Altarelli and G. Parisi, Nucl. Phys. B126 (1977), 298.

[9] T. Gottschalk and D. Sivers, Phys. Rev. D21 (1980), 102;

F. A. Berends, R. Kleiss, P. De Causmaeker, R. Gastmans and T.T. Wu, Phys. Lett. 103B (1981), 124.

[10] S. Parke and T. Taylor, Phys. Rev. Lett. 56 (1986) 2459.

[11] S. Parke and T. Taylor, Nucl. Phys. B269 (1986), 410;

J. Gunion and J. Kalinowski, Phys. Rev. D34 (1986), 2119.

[12] Z. Kunszt, Nucl. Phys. B271 (1986), 333.

[13] M. Mangano and S. Parke, Proc. of the 'International Europhysics Conference on High Energy Physics', Uppsala, Sweden, (1987), ed. O. Botner, p.201.

[14] M. Mangano and S. Parke, Nucl. Phys. B299 (1988) 673.

[15] M. Mangano, Nucl. Phys. B315 (1989) 391.

[16] Z. Kunszt and W. J. Stirling, Phys. Rev. D30 (1988),2439;

C. J. Maxwell, Phys. Lett. 192B (1987), 190;

M. Mangano and S. Parke, Phys. Rev. D39 (1989), 758;

C. J. Maxwell, Nucl. Phys. B316, (1989), 312. 